Revista Calidad en la Educación Superior

Programa de Autoevaluación Académica

Universidad Estatal a Distancia, Costa Rica

ISSN 1659-4703

revistacalidad@uned.ac.cr

\title{
INDICADORES DE DESEMPEÑO PARA INVESTIGACIÓN UNIVERSITARIA, EN EL CONTEXTO DE MEGA PLANEACIÓN.
}

\section{PERFORMANCE INDICATORS FOR UNIVERSITY RESEARCH IN THE CONTEXT OF MEGA PLANNING.}

\author{
José Irigoyen Baldenegro ${ }^{1}$ \\ jirigoyen2002@yahoo.com.mx \\ Instituto Tecnológico de Sonora (ITSON), México
}

Volumen 6, Número 2

Noviembre, 2015

Pp. $113-135$

Recibido: 2 de mayo, 2014

Aprobado: 25 de setiembre, 2015

${ }^{1}$ Instituto Tecnológico de Sonora (ITSON), México. Candidato a Doctor por el Instituto Tecnológico de Sonora (ITSON) 


\section{Resumen}

La competitividad garantiza pertinencia y sustentabilidad a una organización, debiéndose fundamentar en una planeación estratégica tipo mega que garantice el logro de su misión y visión, donde los indicadores constituyen los signos vitales, precisos y válidos sobre el desempeño, identificados en tres niveles de planeación, tendiente a definir y lograr el éxito organizacional: Mega (Estratégico), Macro (Táctico) y Micro (Operacional),partiendo de analizar la contribución social y valor agregado, alineando después metas y resultados de la organización. En este contexto, con base en referentes para el área de evaluación en investigación universitaria y benchmarking ${ }^{2}$ aplicado sobre universidades de excelencia, se propone una selección de indicadores, perfilados a fortalecer el desempeño y control de la función misional de investigación universitaria.

Palabras clave: Planeación estratégica, evaluación institucional, instrumentos de medición, investigación institucional, gestión estratégica.

\section{Abstract}

It ensures competitiveness and sustainability relevance to an organization, having to be based on a strategic planning mega type to ensure the achievement of its mission and vision, where indicators are vital, accurate and valid signs on performance, identified three levels of planning aimed at defining and achieving organizational success: Mega(Strategic), Macro(Tactical) and Micro (Operational); based on analyzing social contribution and value added, after aligning goals and outcomes of the organization. In this context, based on benchmarks for the assessment area in university research and applied benchmarking ${ }^{2}$ universities of excellence, we propose a selection of indicators, outlined to strengthen the performance and control of missionary function of university research.

Keywords: Strategic planning, institutional assessment, measuring instruments, institutional research, strategic management.

\footnotetext{
${ }^{2}$ Benchmarking. "Proceso continuo y sistemático de evaluar los productos, servicios o procesos de las organizaciones que son reconocidas por ser representativas de las mejores prácticas para efectos de mejora organizacional". Spendolini, M.J. http://benchmarking.galeon.com/definicion.html.

${ }^{2}$ Benchmarking. "Continuous and systematic process of evaluating the products, services or processes of organizations that are recognized as being representative of best practices for the purpose of organizational improvement". Spendolini, M.J. http://benchmarking.galeon.com/definicion.html
} 


\section{Introducción.}

A partir de mediados del siglo pasado, las organizaciones han experimentando procesos de cambio significativos, inéditos e impredecibles, en función de la transición desde el proteccionismo hasta la actualidad, determinada por un entorno abierto y sumamente competido. En este contexto, la competitividad constituye el referente clave que garantiza la pertinencia y sustentabilidad de una organización, tanto en su presente como en el futuro. Por lo que el logro organizacional, en términos competitivos, debe estar contextualizado y soportado en un plan estratégico, el cual, fije la visión, misión, objetivos y estrategias, con base en un pertinente análisis y diagnóstico. Enmarcándose el control del plan en una serie de acciones orientadas a medir, evaluar, ajustar y regular las actividades planteadas en él.

En el desarrollo de esta medición, Lezama (2007) establece que los indicadores de gestión se convierten en los signos vitales de toda organización, y su continuo monitoreo, permite establecer las condiciones e identificar los diversos síntomas que se derivan del desarrollo de sus actividades. Adicionando, que en una organización se debe contar con al menos un mínimo de indicadores, que garantice contar con información confiable, precisa y constante, relativa a una serie de referentes de desempeño como: Efectividad, eficiencia, eficacia, productividad, calidad, cobertura, incidencia, entre otras. Constituyendo estos indicadores, el conjunto de signos vitales de la organización.

En el contexto específico para las Instituciones de Educación Superior (IES), estas desde sus orígenes han tenido la misión y visión de generar, difundir y aplicar conocimiento en sus diversas vertientes, tendiente a contribuir a solucionar los retos que cotidianamente enfrentan las diversas regiones de su área de influencia e impacto.

Lo anterior, hace que la investigación universitaria adquiera un carácter estratégico, al mantener una gran responsabilidad en la búsqueda de respuestas y cristalización de soluciones a necesidades sociales concretas. 
Por lo que, el principal reto para las IES, consiste en proveer a la creciente sociedad demandante, de las herramientas, metodologías y avances tecnológicos suficientes y pertinentes, trasladando dichos conocimientos al sector productivo para su transformación en bienes, procesos y servicios útiles, aprovechables por la sociedad.

En este marco, a las IES, de acuerdo con Ortiz (2005) no debe bastarles con ser eficientes, sino también eficaces, requiriendo por lo tanto, diseñar e implementar un plan estratégico que incluya procesos, mecanismos e instrumentos, en términos de productividad, calidad, gestión, organización, evaluación y mejora continua, tendiente a responder suficiente y pertinentemente a los requerimientos planteados por su entorno demandante. Circunstancia indispensable a efecto de lograr su consolidación, reconocimiento y sustentabilidad.

Así, el que las IES operen conforme a un plan estratégico integral les permite: Lograr ventajas competitivas, disminuir riesgos, optimizar recursos, detectar desviaciones, mejorar la calidad, elevar la satisfacción del cliente y expeditar tiempos de respuesta; consolidando la capacidad y éxito institucional para atender eficaz y eficientemente las señales evolutivas de su entorno, en un medio cada vez más hostil y competitivo. De esta manera, el esfuerzo sistematizado y continuo de plantear estratégicamente el horizonte debe de constituir la premisa de las universidades con visión de excelencia.

\section{Contextualización.}

A lo largo de la historia de la administración se han desarrollado métodos e instrumentos para establecer y mejorar las normas de actuación de las instituciones e individuos que las integran, donde las IES no han estado ajenas a estas herramientas. En este contexto, sus perspectivas han estado vinculadas al desarrollo que ha tenido la ciencia de la administración, desde sus inicios con los trabajos de los clásicos Fayol y Taylor (Fayol, 1987), hasta la tendencia más aceptada en la actualidad que es la teoría de sistemas, en la que se ve a toda institución o entidad como un sistema (Ávila, 2015). Circunstancia 
contextualizada por González (2005) al establecer que se utiliza un enfoque sistémico cuando se desarrollan modelos integrales en los que se articulan los sistemas de control interno, gestión de la calidad, desarrollo administrativo y sistemas de medición, y que de acuerdo a González \& Ayarza (1997) en el contexto específico de las IES, estas (Sistema educativo) son un sistema dentro de un sistema más complejo y dinámico (Social) caracterizadas por un proceso de intercambio permanente y constante.

Actualmente, las formas tradicionales de planeación y gestión se han transformado radicalmente y las universidades, en términos de sus funciones sustantivas tradicionales (Docencia, investigación y extensión), tienen un gran reto por delante que sólo pueden enfrentarlo cambiando o fortaleciendo sus modelos de gestión.

Lo anterior impone el que las IES deben adoptar innovadores sistemas de gestión, que incluyan mediciones objetivas y rigurosas de sus procesos, en función de una actuación mas activa, consistente y pertinente, particularmente, las universidades públicas. En este contexto Royero (2003), establece que la realidad de las IES requiere de soluciones viables a los múltiples problemas que confrontan sus procesos básicos, como es el caso de la función de investigación. Misma que tendiente a su dirección eficaz, debe comenzar con una planeación estratégica donde los objetivos y la visión sean la base para la definición de las áreas claves de gestión, con el propósito de definir indicadores y estándares que permitan la evaluación y el control de dichos procesos, tendiente a la satisfacción de los beneficiarios del sistema, en un marco de mejora continua.

Un elemento básico para la derivación de referentes evaluativos útiles, de acuerdo con Guerra (2007), es la estructuración de un listado de objetivos de desempeño, producto de un proceso sobre detección y selección de necesidades, los cuales deben incluir al menos, el logro real a ser observado, los criterios de medición, quién o qué entidad es responsable por dicho logro y las condiciones bajo las cuales el resultado es aceptable. Por definición, el concepto de desempeño (performance) siempre enfoca su atención en la obtención de resultados, considerados como la meta y propósito de las actividades, 
procesos organizacionales y comportamiento individual. Estos resultados deben ser medibles por métodos objetivos y rigurosos, basados en indicadores visibles de comportamiento o productos de dicho comportamiento y utilizados como estándares para orientación, retroalimentación y autocorrección.

Lezama (2007), a su vez, define el desempeño como aquellas acciones que son relevantes para lograr los objetivos de la organización, y que pueden ser medidas en términos de contribución a las metas al constituir un concepto plural que busca englobar diversos factores en un elemento mesurable y cuantificable. Por lo que el desempeño se relaciona en un ámbito estratégico al asociar las metas logradas y los recursos organizativos utilizados, enmarcados en condiciones de exigencia particular, provistos con atributos de calidad.

Complementariamente, Lezama (2007) establece que los indicadores de gestión tienen la finalidad de guiar y controlar el desempeño objetivo y comportamental requerido para el logro de los objetivos, a través de las estrategias organizacionales. Por ello, para medir el desempeño, se necesita evaluarlo a través de indicadores a fin de apoyar a la institución para determinar cuán efectivo y eficiente ha sido el logro de los objetivos y cumplimiento de la metas. Así mismo, Franklin (2001) enfatiza que un indicador constituye un punto que, en una estadística simple o compuesta, refleja algún rasgo importante de un sistema al ser medible, relevante y vinculante.

Por su parte, la Universidad Autónoma de México (UNAM, 2005) especifica que los indicadores son medidas cuantificables para evaluar el desempeño en las dimensiones básicas de un programa o una actividad determinada. En este sentido, la utilización de indicadores estratégicos es parte central del proceso de evaluación y seguimiento institucional, al constituirse como expresiones cuantitativas que reflejan aspectos parciales de fenómenos complejos, muestran relaciones cuantitativas entre dos variables y que para tener validez, deben estar alineados al propósito y objetivos de los planes y programas de desarrollo. 
En el contexto anterior, se observa que la identificación, diseño, procesamiento, monitoreo y análisis de indicadores constituyen actividades esenciales en el proceso de evaluación de los resultados de la gestión organizacional. La sustentación en términos de la consistencia, confiabilidad, validez y eficacia de la presente gestión, estará en función del grado de alineación respecto a sus objetivos estratégicos (áreas críticas) y los Factores Críticos de Éxito (FCE).

En una organización donde todo se integra en un plano de interrelación, Guerra (2007) afirma que cuando se evalúa para determinar si una solución cumple con los objetivos, se debe incluir en este proceso, tanto el cumplimiento de objetivos directos como el objetivo final; esto infiere que un marco de trabajo útil para dicho proceso, lo constituye la mega planeación estratégica (Kaufman, 2005) a través de tres niveles de resultados: Impacto social (expresado mediante una visión ideal, es decir, el nivel estratégico o Mega), logros organizacionales (según lo expresado mediante la misión de la organización, que refiere al nivel táctico o macro), y las entregas internas (usualmente expresadas como metas $u$ objetivos operacionales, identificado como el nivel operacional o micro). Bernárdez (2005), a través de la Metodología del Desempeño Humano o HPT ${ }^{2}$ establece que esta visión ideal mega deberá formularse partiendo de agregar una serie de indicadores sociales, donde la misión de la organización debe estar relacionada y dirigida a lograr mejoras, como forma de garantizar su supervivencia en el largo plazo. Que implementa en este sentido, un proceso de alineación estratégica.

En este contexto Kaufman (2005), especifica que los resultados de desempeño deben ser analizados en tres niveles:

1. Mega: En este nivel se definen los resultados en términos del impacto social deseado; donde los resultados esperados derivan de una visión ideal de la

\footnotetext{
${ }^{2}$ Human Performance Technology (Tecnología del Desempeño Humano). Metodología sistémica para el abordaje integral de la mejora continua y sostenible del desempeño individual, grupal y organizacional. Los principios básicos son: Enfoque sistémico, uso eficiente y efectivo de recursos basado en necesidades reales, enfoque interdisciplinario, y orientación a resultados.
} 
sociedad con base en la cual se definen la misión de la organización y sus integrantes.

Los resultados Mega incluyen aquellos indicadores sociales que impactan en la visión ideal mínima mediante el crecimiento y desarrollo de la sociedad, el mercado y los clientes, estabilidad y progreso social entre otros.

2. Macro: Este nivel refiere a que los resultados son los ingresos y valor generado que benefician a la organización. Se establece en el mercado de la organización con base en diferentes sistemas de medición de su contribución. Un resultado es Macro cuando está definido en términos de su contribución al sostenimiento económico de la organización o a su prestigio y aceptación por parte de sus clientes.

3. Micro: En este nivel, los resultados se miden en términos de los productos internos de la organización, tales como: Productos terminados y en proceso, competencias del personal, desempeño de las personas, calidad de productos y procesos y calidad de insumos y recursos.

Por lo tanto, el análisis y diagnóstico del desempeño en el contexto de la Mega Planeación, involucra el considerar la alineación y efectos en los tres niveles mencionados. Kaufman (2005) hace especial énfasis en medir los resultados de "afuera hacia dentro", partiendo de analizar la contribución social y el valor agregado o entregado y reconocido por los clientes para luego alinear las metas y resultados de la organización y sus integrantes. En este contexto, la prueba final del desempeño estará determinada en función de si cada uno de los niveles estratégico, táctico y operacional (Mega, Macro y Micro) contribuyó o no, y en qué grado, al logro de resultados finales, determinados en una organización por su misión y visión.

En términos relativos a la utilización de la mega planeación estratégica en las instituciones, Kaufman (2005), en una visión integral, la concibe como un sistema que contiene un determinado número de procesos que hacen de ella una actividad dinámica, 
flexible y continua, concebida como un instrumento racionalizador en un entorno cambiante. En este contexto, establece un esquema para su proceso dividido en cinco fases: El proceso comienza identificando las metas o resultados deseados (desempeño deseado), comparándolos con la situación actual. Las diferencias o brechas entre la situación deseada y la actual en términos de objetivos constituyen las necesidades de mejora.

A su vez, Bernárdez (2005) puntualiza que las necesidades deben comenzar a identificarse desde el nivel social o Mega, partiendo de definir una misión basada en la visión ideal de la sociedad, estructurada en términos de indicadores medibles y alcanzables (indicadores-meta sociales), que a su vez permitan definir los organizacionales (Macro) y los atributos de productos o servicios a entregar para lograrlos (Micro).

La siguiente fase es la llamada análisis de causas, en la que se establecen los factores que provocan esas diferencias o brechas y su relación entre sí. La tercera fase se denomina selección y diseño de intervenciones, donde se consideran diversas tecnologías de intervención como medios alternativos o complementarios para resolver los problemas planteados. La cuarta fase es la implementación de la intervención para producir un cambio estable y sostenible en el desempeño, estableciendo un sistema para su gestión. La quinta y última fase consiste en el establecimiento de un subsistema de evaluación, retroalimentación, seguimiento y mejora continua.

En este contexto, la mega planeación estratégica permite la integración de un marco rector, a través del cual, una IES puede normar su criterio para la toma de decisiones asertivas, operando para el caso específico de la actividad investigadora institucional, como una estructura de organización que contribuye al fortalecimiento y mejora continua de los procesos vinculantes a dicha actividad, garantizando la efectividad, consistencia y sinergia de las estrategias y los recursos humanos, materiales y técnicos dedicados a la investigación en términos de mejorar su productividad, calidad e impacto social. 


\section{Estructura.}

La propuesta se genera partir de un plan integral institucional desarrollado para una IES tipo, basada en un Modelo Integral de Gestión y Control de la Investigación (MIGCl), mismo que involucra, en términos generales, los siguientes elementos: Parte de un diagnóstico y de la planeación disponible institucional, consistentes con su misión y visión en términos de mega planeación. En segundo término, se identifican las áreas críticas que delimitan el éxito de la actividad investigadora, así como las variables y procesos involucrados. Posteriormente se establecen una serie de indicadores referenciados por Áreas Críticas de Éxito (ACE) y Factores Críticos de Éxito (FCE). Adicionalmente se implementan instrumentos específicos de control y medición, que permiten el control, evaluación y planeación cíclica de las ACE y FCE. Por último, se definen mecanismos e instrumentos para la presentación de informes periódicos tendiente a la toma de decisiones.

EI MIGCI, funge como un instrumento rector institucional a través del cual se garantice que las estrategias, acciones y recursos, sean eficaz y eficientemente dirigidos hacia los aspectos claves del éxito de la productividad investigadora, satisfacción de los usuarios y el logro de un aporte y valor social.

Con base en lo anterior, generado el diagnóstico integral y alineada la planeación institucional disponible consistentemente con su misión y visión en términos de mega planeación, se procede a la identificación de las áreas y factores críticos de éxito institucional en términos de su actividad investigadora.

Las ACE constituyen el elemento clave para la ejecución del control de gestión, en los términos esperados. La identificación de éstas, debe sustentarse a través de un mapa de procesos, elaborado específicamente para la institución, lo que permite identificar el nivel operativo del sistema a controlar a través del análisis de las actividades de transformación institucionales (insumos en productos y/o servicios institucionales). Es decir, los procesos estratégicos clave y de soporte institucionales (Cadena de valor). 
En este contexto, el proceso clave institucional lo constituye el desarrollar la investigación, innovadora, significativa y de calidad. Este proceso se identifica como el área crítica general, objeto de abordaje a través de la presente propuesta. En forma específica, este proceso, deriva a su vez en las siguientes áreas críticas, vinculantes con los objetivos estratégicos institucionales: Productos de investigación, gestión, desarrollo de investigadores, difusión y divulgación y financiamiento.

Identificadas las ACE, se procede a determinar lo que se pretende controlar en dichas áreas; es decir, los FCE, que enfoca el control de la gestión en los procesos críticos institucionales para el área de investigación. Es decir, en los aspectos vitales que garantizan el éxito de su visión: Eficacia, eficiencia, efectividad, cobertura, pertinencia, impacto, calidad, etc. mismos que, una vez validados, permiten el siguiente paso referido a la incorporación de indicadores clave (vinculables a las metas fijadas) y la asignación de responsables a través de la estructura establecida o en su caso propuesta.

La identificación, diseño, procesamiento, monitoreo y análisis de indicadores, circunscritos en el marco de las ACE y los FCE, constituyen actividades esenciales en el proceso de evaluación y mejora continua, donde su función es medir los resultados de la gestión institucional.

Mediante estos indicadores, se pretende que en lo general sirvan de instrumentos para evaluar hasta qué punto se están logrando los objetivos estratégicos institucionales. También, se procura que estos indicadores representen una unidad de medida para evaluar el desempeño de la institución frente a sus metas compromiso, produzcan información para analizar el desempeño y cumplimiento de objetivos en términos de resultados, detecten y prevean desviaciones en el logro de los objetivos y, generen alertas sobre la acción para mantener la dirección alineada con respecto al plan estratégico establecido. Asimismo, impulsen los esfuerzos para medir los resultados en el abatimiento de los principales rezagos institucionales y el impacto social de las 
acciones realizadas en el ámbito de la investigación, así como disponer de información válida para apoyar la toma de decisiones con un sentido estratégico.

Estructurados los indicadores, se procesa su control a través de dos instrumentos. Se asigna para cada ACE un Cuadro de Control de Mando (CCM) que permitirá monitorear los avances del comportamiento de los resultados de gestión, donde este instrumento, integra contextualmente la relación de indicadores establecidos por cada una de las ACE identificadas para la institución. Incorpora el objetivo, estrategia a que corresponde y el responsable de su monitoreo y resultados, así como la relación de indicadores que se vinculan con un área crítica en particular, revelando su situación actual y perspectiva de logro (meta). Adicionalmente la implementación de un instrumento denominado Cuadro de Mando Integral (CMI) permite visualizar el avance real de los resultados, luego de la frecuencia definida. Identifica la relación de FCE establecidos por cada una de las ACE seleccionadas para la institución, exponiendo la situación actual de los mismos, su perspectiva de logro (meta), así como el grado de cumplimiento que se obtuvo para un período específico.

En la estructuración de la presente propuesta de indicadores, se consideraron lineamientos y criterios establecidos por organismos e instituciones mexicanos, reconocidos en el ámbito de evaluación de la investigación universitaria, tales como la Secretaría de Educación Pública (SEP) a través del Programa Integral de Fortalecimiento Institucional (PIFI) y el Programa para el Desarrollo Profesional Docente para el tipo superior (PRODEP), así como el Sistema Nacional de Investigadores (SNI) a través del Consejo Nacional de Ciencia y Tecnología (CONACYT). Adicionalmente, se integraron indicadores, extrapolados del catálogo de indicadores de desempeño de entidades y dependencias universitarias de la UNAM (2005), así como indicadores de diversas IES, reconocidas en México por su excelencia y alto desempeño en investigación a través de Ranking de Producción Científica Mexicana, promovido por el Foro Consultivo Científico y Tecnológico, AC.(2011). 
El listado de indicadores propuesto, mantiene como objetivo general el coadyuvar en la implementación y/o fortalecimiento del sistema de control para la función misional (sustantiva) de investigación en una IES. El listado mantiene un carácter propositivo y perfectible, en función de lograr la máxima eficacia, eficiencia y pertinencia en la medición del cumplimiento de acciones vinculadas a objetivos institucionales específicos en materia de investigación universitaria. En este sentido, adicionalmente se presenta una relación (causa-efecto) entre los resultados esperados, en términos de objetivos estratégicos y los indicadores de medición requeridos para evaluar efectivamente dichos resultados.

En la Tabla 1, se muestra el listado de indicadores de desempeño propuesto, correlacionados y consistentes con la integración estratégica del sistema de investigación institucional para una IES. En este sentido, las ACE de correspondencia para los indicadores, son cinco: Gestión, productos de investigación, difusión y divulgación, desarrollo de investigadores y financiamiento. Cada una de ellas, se indexa a los FCE, según corresponda; siendo: Eficacia, eficiencia, Efectividad, cobertura, pertinencia, equidad, impacto, productividad, disponibilidad de recursos y calidad. Adicionalmente, se ubica respecto al nivel del elemento organizacional al que pertenece: Mega, macro o micro, así como el objetivo estratégico del que se desprende, culminando con la descripción del indicador y su correspondiente fórmula para su cálculo.

La propuesta, consta de un total de 35 indicadores, los cuáles, de acuerdo con su nivel de clasificación, se organizan de la siguiente forma: 3 indicadores pertenecen al nivel mega, 15 indicadores corresponden al nivel macro y 17 indicadores al nivel micro. Asimismo, en función del área crítica a la que pertenecen, los indicadores se estructuran de la siguiente manera: 11 son indicadores correlacionados a la productividad investigadora institucional; 14 son indicadores atribuibles a la gestión investigadora, 6 corresponden al financiamiento de la investigación institucional, 2 a elementos relacionados con la difusión y divulgación de los productos y servicios de investigación, 
Indicadores de desempeño para investigación universitaria, en el contexto de mega planeación. José Irigoyen Baldenegro

innovación y transferencia de tecnología, y por último, 2 se encuentran indexados a referentes sobre el desarrollo de investigadores. 
Tabla 1. Indicadores de desempeño para para la función misional de investigación en una IES; estructurados en el contexto de la mega planeación.

\begin{tabular}{|c|c|c|c|c|c|c|}
\hline $\begin{array}{lr}\text { Área } & \text { Crítica } \\
\text { de } & \text { Éxito } \\
(\mathrm{ACE}) & \end{array}$ & $\begin{array}{lr}\text { Factor } & \text { crítico } \\
\text { de } & \text { Éxito } \\
(\mathrm{FCE}) & \end{array}$ & $\begin{array}{l}\text { Nivel de } \\
\text { planeación }\end{array}$ & Objetivo estratégico & Indicador & Descripción & Fórmula/Documento \\
\hline Gestión & Calidad & Mega & $\begin{array}{l}\text { Mejorar la calidad de vida de la } \\
\text { sociedad }\end{array}$ & $\begin{array}{l}\text { Satisfacción de } \\
\text { usuarios }\end{array}$ & $\begin{array}{l}\text { Porcentaje de usuarios satisfechos } \\
\text { con servicio y trato del sistema de } \\
\text { investigación }\end{array}$ & Cuestionario \\
\hline $\begin{array}{l}\text { Productos de } \\
\text { investigación }\end{array}$ & Impacto & Mega & $\begin{array}{l}\text { Contribuir a la educación y } \\
\text { conocimiento de la comunidad } \\
\text { a la que sirve la institución }\end{array}$ & $\begin{array}{ll}\text { 2. } & \text { Desarrollo del } \\
\text { conocimiento } & \\
\text { humano } & \end{array}$ & $\begin{array}{l}\text { Promedio de productos de } \\
\text { investigación aplicada realizados con } \\
\text { relación al total de investigadores }\end{array}$ & $\begin{array}{l}\text { Total productos de investigación } \\
\text { básica/Total de investigadores }\end{array}$ \\
\hline $\begin{array}{l}\text { Productos de } \\
\text { investigación }\end{array}$ & Impacto & Mega & $\begin{array}{l}\text { Mejorar la calidad de vida de la } \\
\text { sociedad }\end{array}$ & $\begin{array}{ll}\text { 3. } & \text { Desarrollo } \\
\text { problemática } \\
\text { social }\end{array}$ & $\begin{array}{l}\text { Promedio de productos de } \\
\text { investigación social realizados con } \\
\text { relación al total de investigadores }\end{array}$ & $\begin{array}{l}\text { Total productos de investigación } \\
\text { social/Total de investigadores }\end{array}$ \\
\hline Gestión & $\begin{array}{l}\text { Pertinencia- } \\
\text { eficiencia }\end{array}$ & Macro & $\begin{array}{l}\text { Posicionar a la institución como } \\
\text { referente de investigación } \\
\text { académica }\end{array}$ & $\begin{array}{l}\text { 4. Posicionamiento } \\
\text { nacional de IES }\end{array}$ & $\begin{array}{l}\text { Estudio sobre posicionamiento de las } \\
\text { IES nacionales en materia de } \\
\text { productividad y competitividad } \\
\text { investigadora. }\end{array}$ & Documento-informe \\
\hline Gestión & $\begin{array}{l}\text { Impacto- } \\
\text { eficiencia }\end{array}$ & Macro & $\begin{array}{l}\text { Incrementar y consolidar la } \\
\text { satisfacción de los usuarios de } \\
\text { los productos y servicios de } \\
\text { investigación }\end{array}$ & $\begin{array}{ll}5 . & \text { Imagen } \\
\text { institucional en la } \\
\text { sociedad }\end{array}$ & $\begin{array}{l}\text { Estudio sobre imagen institucional en } \\
\text { materia de referencia, impacto y } \\
\text { productividad investigadora. }\end{array}$ & Documento-informe \\
\hline Gestión & $\begin{array}{l}\text { Pertinencia- } \\
\text { eficiencia }\end{array}$ & Macro & $\begin{array}{l}\text { Incrementar y consolidar la } \\
\text { satisfacción de los actores } \\
\text { internos relacionados a la } \\
\text { investigación }\end{array}$ & $\begin{array}{ll}\text { 6. } & \begin{array}{l}\text { Satisfacción de } \\
\text { investigadores }\end{array}\end{array}$ & $\begin{array}{l}\text { Estudio sobre imagen institucional en } \\
\text { la planta investigadora en materia de } \\
\text { referencia, impacto y productividad } \\
\text { de investigación }\end{array}$ & Documento-informe \\
\hline Gestión & $\begin{array}{l}\text { Eficiencia- } \\
\text { pertinencia }\end{array}$ & Macro & $\begin{array}{l}\text { Incrementar y consolidar la } \\
\text { vinculación significativa para la } \\
\text { investigación institucional }\end{array}$ & $\begin{array}{ll}\text { 7. } & \text { Movilidad de } \\
\text { investigadores } \\
\text { institucionales }\end{array}$ & $\begin{array}{l}\text { Total de movilidad interinstitucional } \\
\text { de investigadores durante el año }\end{array}$ & $\begin{array}{l}\text { Total de investigadores que desarrollaron } \\
\text { movilidad/ Total de investigadores * } 100\end{array}$ \\
\hline $\begin{array}{l}\text { Productos de } \\
\text { investigación }\end{array}$ & Eficiencia & Macro & $\begin{array}{l}\text { Disponer de } \begin{array}{r}\text { cuerpos } \\
\text { suficientes, }\end{array} \\
\text { académicos } \\
\text { pertinentes y reconocidos }\end{array}$ & $\begin{array}{l}\text { Cuerpos } \\
\text { académicos }\end{array}$ & $\begin{array}{l}\text { Total de cuerpos académicos } \\
\text { institucionales (CAEF: Cuerpos } \\
\text { académicos en formación; CAEC: } \\
\text { Cuerpos académicos } r \text { en } \\
\text { consolidación, CAC: Cuerpos } \\
\text { académicos consolidados) }\end{array}$ & $\begin{array}{l}\text { Total de CAEF/Total de } \mathrm{CA}^{*} 100 \\
\text { Total de CAEC/Total } \mathrm{CA}^{*} 100 \\
\text { Total CAC/Total } \mathrm{CA}^{*} 100 \\
\text { Total CA/Total CA proyectados año } \\
\text { referencia * } 100\end{array}$ \\
\hline $\begin{array}{l}\text { Productos de } \\
\text { investigación }\end{array}$ & Eficiencia & Macro & $\begin{array}{l}\text { Incrementar la formación y } \\
\text { adherencia de redes de } \\
\text { investigación significativas }\end{array}$ & $\begin{array}{ll}9 . & \begin{array}{l}\text { Redes } \\
\text { investigación }\end{array}\end{array}$ & $\begin{array}{l}\text { Total de redes de investigación de } \\
\text { auto creación institucional y externas }\end{array}$ & $\begin{array}{l}\text { Total de redes de investigación propias/ } \\
\text { Total de redes de investigación } \\
\text { institucional }{ }^{*} 100\end{array}$ \\
\hline
\end{tabular}


Indicadores de desempeño para investigación universitaria, en el contexto de mega planeación. José Irigoyen Baldenegro

\begin{tabular}{|c|c|c|c|c|c|c|}
\hline $\begin{array}{lr}\text { Área } & \text { Crítica } \\
\text { de } & \text { Éxito } \\
(\mathrm{ACE}) & \end{array}$ & $\begin{array}{lr}\text { Factor } & \text { crítico } \\
\text { de } & \text { Éxito } \\
\text { (FCE) } & \end{array}$ & $\begin{array}{l}\text { Nivel de } \\
\text { planeación }\end{array}$ & Objetivo estratégico & Indicador & Descripción & Fórmula/Documento \\
\hline & & & & & & $\begin{array}{l}\text { Total de redes de investigación externas/ } \\
\text { Total de redes de investigación } \\
\text { institucional* } 100\end{array}$ \\
\hline $\begin{array}{l}\text { Productos de } \\
\text { investigación }\end{array}$ & Eficiencia & Macro & $\begin{array}{l}\text { Incrementar y consolidar la } \\
\text { planta investigadora }\end{array}$ & 10. Investigadores & $\begin{array}{l}\text { Porcentaje de investigadores } \\
\text { respecto al total de PTC }\end{array}$ & Total de investigadores/Total de PTC * 100 \\
\hline $\begin{array}{l}\text { Productos de } \\
\text { investigación }\end{array}$ & Eficiencia & Macro & $\begin{array}{l}\text { Incorporar y mantener a los } \\
\text { investigadores institucionales } \\
\text { como miembros del Sistema } \\
\text { Nacional de Investigadores } \\
\text { (SIN) }\end{array}$ & $\begin{array}{l}\text { 11. Investigadores en } \\
\text { SNI }\end{array}$ & $\begin{array}{l}\text { Porcentaje de investigadores } \\
\text { en el SNI, respecto al total de } \\
\text { investigadores }\end{array}$ & $\begin{array}{l}\text { Investigadores en SNI/total investigadores * } \\
100\end{array}$ \\
\hline $\begin{array}{l}\text { Productos de } \\
\text { investigación }\end{array}$ & & Macro & $\begin{array}{l}\text { Incrementar y consolidar la } \\
\text { satisfacción de los usuarios de } \\
\text { los productos y servicios de } \\
\text { investigación institucionales }\end{array}$ & $\begin{array}{l}\text { 12. Cumplimiento de } \\
\text { proyectos }\end{array}$ & $\begin{array}{l}\text { Porcentaje de proyectos } \\
\text { Terminados en el año de referencia } \\
\text { respecto a los programados. }\end{array}$ & $\begin{array}{l}\text { Proyectos terminados en año N/Proyectos } \\
\text { programados terminar en año } N * 100\end{array}$ \\
\hline $\begin{array}{l}\text { Productos de } \\
\text { investigación }\end{array}$ & Eficiencia & Macro & $\begin{array}{l}\text { Incrementar la cantidad, } \\
\text { calidad, impacto social y } \\
\text { satisfacción de usuarios de los } \\
\text { productos, servicios de } \\
\text { investigación y transferencia de } \\
\text { tecnología institucionales. }\end{array}$ & $\begin{array}{l}\text { 13. } \begin{array}{l}\text { Cumplimiento } \\
\text { artículos }\end{array} \\
\text { publicados en } \\
\text { revistas }\end{array}$ & $\begin{array}{l}\text { Porcentaje de artículos } \\
\text { publicados por tipo de revista (1. con } \\
\text { arbitraje e indizadas, en } \\
\text { formato impreso o electrónico } \\
\text { 2. no indizadas, en formato } \\
\text { impreso o electrónico) } \\
\text { en el año, respecto al total de } \\
\text { artículos programados publicar }\end{array}$ & $\begin{array}{l}\text { Artículos publicados por tipo de revista en } \\
\text { año } N / \text { Artículos programados publicar en } \\
\text { año } N * 100\end{array}$ \\
\hline $\begin{array}{l}\text { Productos de } \\
\text { investigación }\end{array}$ & $\begin{array}{l}\text { Eficiencia- } \\
\text { Impacto }\end{array}$ & Macro & $\begin{array}{l}\text { Incrementar la cantidad de } \\
\text { patentes y marcas, indexadas } \\
\text { a los productos y servicios } \\
\text { institucionales de investigación. }\end{array}$ & $\begin{array}{l}\text { 14. Otros productos } \\
\text { de investigación }\end{array}$ & $\begin{array}{l}\text { Porcentaje de otros productos de } \\
\text { investigación (Patentes, marcas, } \\
\text { etc.) }\end{array}$ & $\begin{array}{l}\sum \text { otros productos de investigación/Total de } \\
\text { productos de investigación * } 100\end{array}$ \\
\hline $\begin{array}{l}\text { Productos de } \\
\text { investigación }\end{array}$ & $\begin{array}{l}\text { Eficiencia- } \\
\text { Impacto }\end{array}$ & Macro & $\begin{array}{l}\text { Incrementar la cantidad, } \\
\text { calidad, impacto social y } \\
\text { satisfacción de usuarios de } \\
\text { productos de investigación. } \\
\text { Ampliación y consolidación de } \\
\text { mercados metas de productos } \\
\text { y servicios de investigación. }\end{array}$ & $\begin{array}{l}\text { 15. } \begin{array}{l}\text { Proyectos } \\
\text { innovación }\end{array} \\
\text { tecnológica } \\
\text { transferencia de } \\
\text { tecnología }\end{array}$ & $\begin{array}{lrr}\text { Porcentaje de proyectos } & \text { de } \\
\text { innovación tecnológica } & \text { y } \\
\text { transferencia de tecnología en } & \\
\text { el año, respecto al total de } & \\
\text { proyectos } & \end{array}$ & $\begin{array}{l}\text { Proyectos de transferencia de tecnología y } \\
\text { transferencia de tecnología para } \\
\text { el sector público o el sector social/ } \\
\text { Total de proyectos * } 100\end{array}$ \\
\hline $\begin{array}{l}\text { Difusión y } \\
\text { divulgación }\end{array}$ & Eficiencia & Macro & $\begin{array}{l}\text { Incrementar la cantidad, } \\
\text { calidad, e impacto social de los } \\
\text { productos, servicios y }\end{array}$ & $\begin{array}{llr}\text { 16. } & \text { Promedio } & \text { de } \\
\text { publicaciones } & \text { de } \\
\text { divulgación } & \text { por }\end{array}$ & $\begin{array}{l}\text { Promedio de publicaciones de } \\
\text { divulgación (libros, artículos } \\
\text { en periódicos o revistas en }\end{array}$ & $\begin{array}{l}\text { Publicaciones de } \\
\text { divulgación por investigador/ } \\
\text { Total de investigadores }\end{array}$ \\
\hline
\end{tabular}


Indicadores de desempeño para investigación universitaria, en el contexto de mega planeación.

José Irigoyen Baldenegro

\begin{tabular}{|c|c|c|c|c|c|c|c|}
\hline $\begin{array}{lr}\text { Area } & \text { Crítica } \\
\text { de } & \text { Éxito } \\
(\mathrm{ACE}) & \end{array}$ & $\begin{array}{ll}\text { Factor } & \text { crítico } \\
\text { de } & \text { Éxito } \\
(\mathrm{FCE}) & \end{array}$ & $\begin{array}{l}\text { Nivel de } \\
\text { planeación }\end{array}$ & Objetivo estratégico & & Indicador & Descripción & Fórmula/Documento \\
\hline & & & $\begin{array}{l}\text { transferencia de tecnología } \\
\text { institucionales. }\end{array}$ & & investigador & $\begin{array}{l}\text { cualquier soporte) por investigadores } \\
\text { en el año }\end{array}$ & \\
\hline Financiamiento & $\begin{array}{l}\text { Eficiencia- } \\
\text { Disponibilidad } \\
\text { de recursos }\end{array}$ & Macro & $\begin{array}{l}\text { Ampliación y consolidación de } \\
\text { mercados metas de productos } \\
\text { y servicios de investigación }\end{array}$ & 17. & $\begin{array}{lr}\text { Ingresos } & \text { por } \\
\text { ventas } & \text { de } \\
\text { productos } & y \\
\text { servicios } & \\
\text { institucionales } & \text { de } \\
\text { investigación } & \end{array}$ & $\begin{array}{l}\text { Porcentaje de ingresos por venta de } \\
\text { productos y servicios, derivados de } \\
\text { la acción investigadora institucional }\end{array}$ & $\begin{array}{l}\text { Total de ventas por productos } \\
\text { institucionales de investigación/ total de } \\
\text { ingresos propios institucionales * } 100\end{array}$ \\
\hline Financiamiento & Eficiencia & Macro & 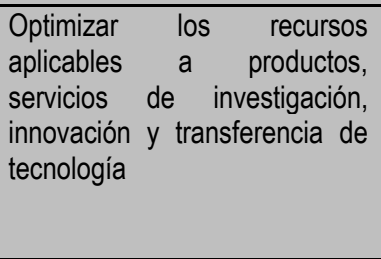 & 18. & $\begin{array}{l}\text { Proyectos con } \\
\text { financiamiento } \\
\text { interno y externo }\end{array}$ & $\begin{array}{l}\text { Porcentaje de proyectos } \\
\text { de investigación con } \\
\text { financiamiento de recursos que } \\
\text { destina la IES y externos }\end{array}$ & $\begin{array}{l}\text { Proyectos de investigación con } \\
\text { financiamiento interno/ Total de proyectos } \\
\text { de investigación * } 100 \\
\text { Proyectos de investigación con } \\
\text { financiamiento externo/ Total de proyectos } \\
\text { de investigación * } 100\end{array}$ \\
\hline $\begin{array}{l}\text { Productos de } \\
\text { investigación }\end{array}$ & Eficiencia & Micro & $\begin{array}{l}\text { Incrementar la cantidad, } \\
\text { calidad, impacto social y } \\
\text { vinculación de los productos y } \\
\text { servicios de investigación. } \\
\text { Optimizar los recursos } \\
\text { aplicables a productos y } \\
\text { servicios de investigación, }\end{array}$ & 19. & $\begin{array}{l}\text { Proyectos } \\
\text { interinstitucionales }\end{array}$ & $\begin{array}{l}\text { Porcentaje de proyectos de } \\
\text { investigación en los que } \\
\text { participan instituciones } \\
\text { diferentes a la IES }\end{array}$ & $\begin{array}{l}\text { Proyectos de } \quad \text { investigación } \\
\text { interinstitucionales/Total } \\
\text { investigación * } 100\end{array}$ \\
\hline Gestión & $\begin{array}{l}\text { Cobertura- } \\
\text { Pertinencia }\end{array}$ & Micro & $\begin{array}{l}\text { Incorporar y mantener al total } \\
\text { de la planta investigadora con } \\
\text { el perfil PRODEP }\end{array}$ & 20. & $\begin{array}{l}\text { Investigadores } \\
\text { perfil PRODEP }\end{array}$ & $\begin{array}{l}\text { Porcentaje de investigadores } \\
\text { Con perfil PRODEP, respecto al total } \\
\text { de investigadores }\end{array}$ & $\begin{array}{l}\text { No. Investigadores perfil PRODEP/total } \\
\text { investigadores * } 100\end{array}$ \\
\hline Gestión & Eficiencia & Micro & $\begin{array}{l}\text { Incrementar la cantidad, } \\
\text { calidad, impacto social y } \\
\text { satisfacción de usuarios de los } \\
\text { productos y servicios de } \\
\text { investigación. Posicionar a la } \\
\text { institución como referente de } \\
\text { investigación académica } \\
\text { nacional }\end{array}$ & 21. & $\begin{array}{lr}\text { Arbitraje } & \text { de } \\
\text { publicaciones } & y \\
\text { evaluación } & \text { de } \\
\text { proyectos } & \end{array}$ & $\begin{array}{l}\text { Porcentaje de investigadores que } \\
\text { participan en el arbitraje de } \\
\text { publicaciones y evaluación de } \\
\text { proyectos }\end{array}$ & $\begin{array}{l}\text { Total de investigadores } \\
\text { participantes en arbitraje de } \\
\text { publicaciones y evaluación de proyectos/ } \\
\text { total de investigadores * } 100\end{array}$ \\
\hline Gestión & Eficiencia & Micro & $\begin{array}{l}\text { Posicionar a la institución como } \\
\text { referente de investigación } \\
\text { académica nacional. } \\
\text { Incrementar la cantidad, }\end{array}$ & 22. & $\begin{array}{lr}\text { Promedio } & \text { de } \\
\text { trabajos de } & \text { de } \\
\text { titulación dirigidos } \\
\text { por investigadores }\end{array}$ & $\begin{array}{l}\text { Promedio de trabajos de } \\
\text { titulación (tesis, tesinas, } \\
\text { etcétera, de cualquier nivel de } \\
\text { estudios), bajo la dirección de }\end{array}$ & $\begin{array}{l}\sum \text { Trabajos de titulación } \\
\text { dirigidos por investigadores/ } \\
\text { Total de investigadores }\end{array}$ \\
\hline
\end{tabular}


Indicadores de desempeño para investigación universitaria, en el contexto de mega planeación. José Irigoyen Baldenegro

\begin{tabular}{|c|c|c|c|c|c|c|}
\hline $\begin{array}{lr}\text { Área } & \text { Crítica } \\
\text { de } & \text { Éxito } \\
(\mathrm{ACE}) & \end{array}$ & $\begin{array}{lr}\text { Factor } & \text { crítico } \\
\text { de } & \text { Éxito } \\
\text { (FCE) } & \end{array}$ & $\begin{array}{l}\text { Nivel de } \\
\text { planeación }\end{array}$ & Objetivo estratégico & Indicador & Descripción & Fórmula/Documento \\
\hline & & & $\begin{array}{l}\text { calidad e impacto social de los } \\
\text { productos y servicios de } \\
\text { investigación }\end{array}$ & & investigadores en el año & \\
\hline Gestión & Eficiencia & Micro & $\begin{array}{l}\text { Posicionar a la institución como } \\
\text { referente de investigación } \\
\text { académica nacional. } \\
\text { Incrementar la cantidad, } \\
\text { calidad e impacto social de los } \\
\text { productos y servicios de } \\
\text { investigación }\end{array}$ & $\begin{array}{l}\text { 23. Investigadores } \\
\text { que participan en } \\
\text { comités tutoriales }\end{array}$ & $\begin{array}{l}\text { Porcentaje de investigadores que } \\
\text { participan en comités tutoriales } \\
\text { en el año }\end{array}$ & $\begin{array}{l}\text { Total de investigadores que } \\
\text { participan en comités tutoriales/ } \\
\text { Total de investigadores * } 100\end{array}$ \\
\hline Gestión & $\begin{array}{l}\text { Eficiencia- } \\
\text { Impacto }\end{array}$ & Micro & \begin{tabular}{lcc} 
Consolidar la & \multicolumn{2}{c}{ vinculación } \\
significativa & para & la \\
investigación institucional &
\end{tabular} & $\begin{array}{ll}\text { 24. Cumplimiento } & \text { de } \\
\text { acciones } & \text { de } \\
\text { vinculación } & \end{array}$ & $\begin{array}{l}\text { Porcentaje de acciones de } \\
\text { vinculación con diversos sectores } \\
\text { realizadas en el año, } \\
\text { respecto a las programadas }\end{array}$ & $\begin{array}{l}\text { Total de acciones de } \\
\text { vinculación académica realizadas/ } \\
\text { Total de acciones de vinculación } \\
\text { académica programadas * } 100\end{array}$ \\
\hline Gestión & Eficiencia & Micro & $\begin{array}{l}\text { Consolidar la vinculación. } \\
\text { Ampliación y consolidación de } \\
\text { mercados metas de productos } \\
\text { y servicios de investigación }\end{array}$ & $\begin{array}{l}\text { 25. Convenios } \\
\text { realizados }\end{array}$ & $\begin{array}{l}\text { Porcentaje de convenios } \\
\text { realizados en el año, } \\
\text { respecto a los programados a } \\
\text { realizar }\end{array}$ & $\begin{array}{l}\text { Total de convenios realizados/ } \\
\text { Total de convenios programados realizar * } \\
100\end{array}$ \\
\hline Gestión & $\begin{array}{l}\text { Cobertura- } \\
\text { Eficiencia }\end{array}$ & Micro & $\begin{array}{l}\text { Incrementar y consolidar la } \\
\text { satisfacción de los usuarios de } \\
\text { los productos y servicios de } \\
\text { investigación institucionales }\end{array}$ & $\begin{array}{ll}26 . & \text { Procesos } \\
\text { certificados en } \\
\text { investigación }\end{array}$ & $\begin{array}{l}\text { Porcentaje de procesos } \\
\text { certificados con } \\
\text { respecto al total de procesos } \\
\text { administrativos institucionales }\end{array}$ & $\begin{array}{l}\begin{array}{l}\text { Procesos de } \\
\text { certificados/Total } \\
\text { administrativos * } 100\end{array} \\
\begin{array}{l}\text { Total de procesos de investigación } \\
\text { procesos }\end{array} \\
\text { certificados/ Total de procesos de } \\
\text { investigación * 100 }\end{array}$ \\
\hline Gestión & $\begin{array}{l}\text { Cobertura- } \\
\text { Eficiencia }\end{array}$ & Micro & $\begin{array}{l}\text { Incrementar y fortalecer la } \\
\text { normativa y procedimientos } \\
\text { certificados aplicables a la } \\
\text { investigación institucional. }\end{array}$ & $\begin{array}{ll}\text { 27. } & \text { Desarrollo de } \\
\text { Normativa } & \end{array}$ & $\begin{array}{l}\text { Promedio de normativa desarrollada } \\
\text { y actualizada para investigación } \\
\text { respecto a la normativa desarrollada } \\
\text { y actualizada institucionalmente }\end{array}$ & $\begin{array}{l}\sum \text { de normativa nueva y actualizada de } \\
\text { investigación/ total de normativa nueva y } \\
\text { actualizada institucional } \\
\sum \text { de normativa nueva y actualizada de } \\
\text { investigación aprobada/ total de iniciativas } \\
\text { sobre normativa nueva y actualizada de } \\
\text { investigación propuesta para autorización } \\
\text { durante año } N^{*} 100\end{array}$ \\
\hline Gestión & $\begin{array}{l}\text { Calidad- } \\
\text { Eficiencia } \\
\text { Pertinencia }\end{array}$ & Micro & $\begin{array}{l}\text { Consolidar el sistema } \\
\text { estratégico de gestión y control } \\
\text { de la actividad investigadora en }\end{array}$ & $\begin{array}{l}\text { 28. Observaciones y } \\
\text { recomendaciones }\end{array}$ & $\begin{array}{l}\text { Promedio de observaciones y } \\
\text { recomendaciones emitidas para } \\
\text { investigación por el órgano de }\end{array}$ & $\begin{array}{l}\Sigma \text { de observaciones y recomendaciones } \\
\text { emitidas por el OCGI para investigación/ } \\
\text { Total de observaciones y recomendaciones }\end{array}$ \\
\hline
\end{tabular}


Indicadores de desempeño para investigación universitaria, en el contexto de mega planeación. José Irigoyen Baldenegro

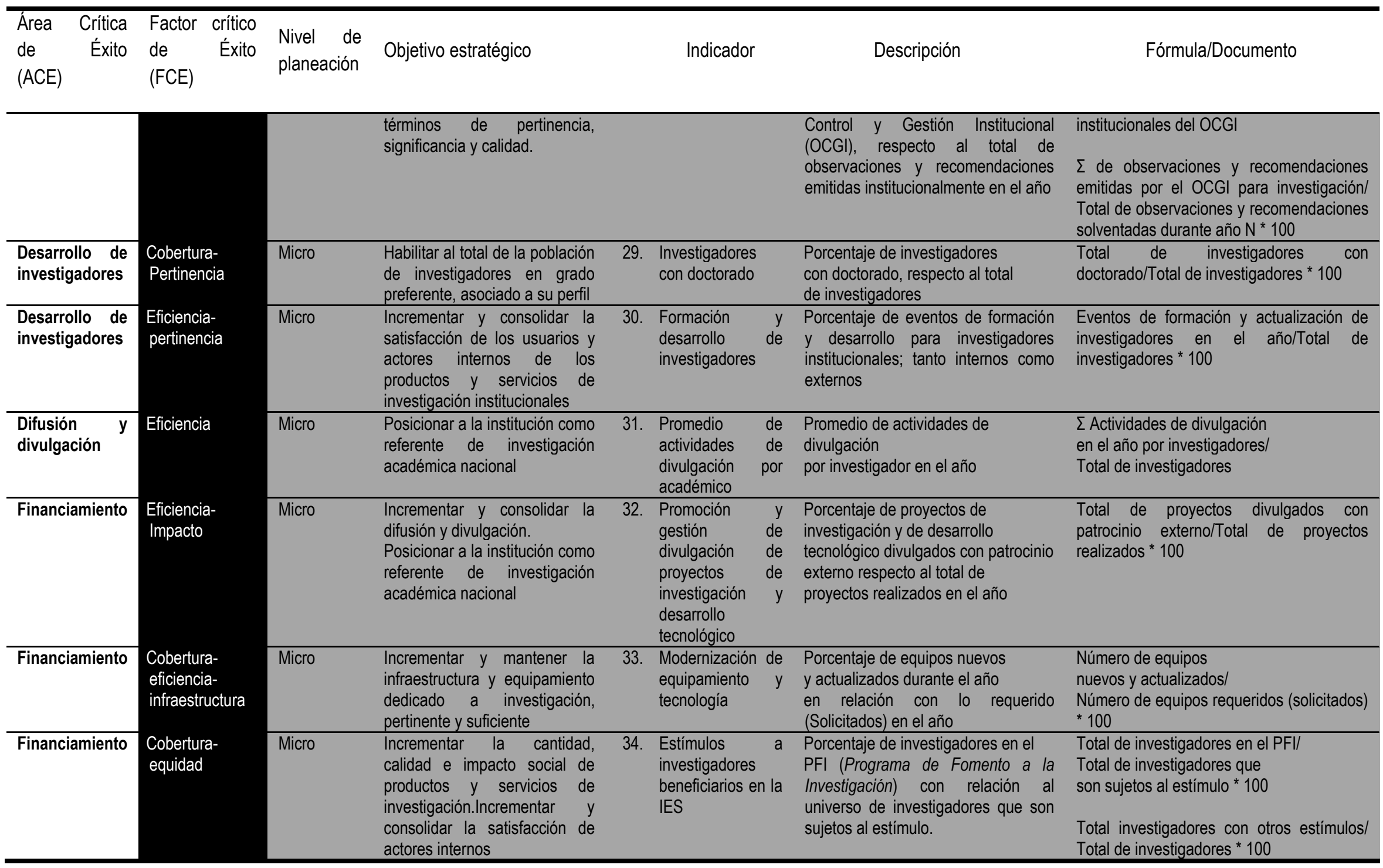


Indicadores de desempeño para investigación universitaria, en el contexto de mega planeación. José Irigoyen Baldenegro

\begin{tabular}{|c|c|c|c|c|c|c|}
\hline $\begin{array}{lr}\text { Area } & \text { Crítica } \\
\text { de } & \text { Éxito } \\
(\mathrm{ACE}) & \end{array}$ & $\begin{array}{lr}\text { Factor } & \text { crítico } \\
\text { de } & \text { Éxito } \\
(\mathrm{FCE}) & \end{array}$ & $\begin{array}{l}\text { Nivel de } \\
\text { planeación }\end{array}$ & Objetivo estratégico & Indicador & Descripción & Fórmula/Documento \\
\hline Financiamiento & $\begin{array}{l}\text { Disponibilidad } \\
\text { recursos } \\
\text { (Infraestructura- } \\
\text { financiamiento) }\end{array}$ & Micro & $\begin{array}{l}\text { Incrementar y mantener la } \\
\text { infraestructura y equipamiento } \\
\text { dedicado a investigación, } \\
\text { pertinente y suficiente }\end{array}$ & $\begin{array}{l}\text { 35. Espacios nuevos } \\
\text { dedicados a la } \\
\text { investigación }\end{array}$ & $\begin{array}{l}\text { Porcentaje de recursos } \\
\text { destinados a obra nueva dedicada a } \\
\text { investigación con respecto al total de } \\
\text { recursos asignados por año para } \\
\text { desarrollo de infraestructura en la } \\
\text { IES. }\end{array}$ & $\begin{array}{l}\text { Recursos destinados a obra nueva } \\
\text { dedicada a investigación/ } \\
\text { Total de recursos asignables en año } \mathrm{N} \text { en } \\
\text { infraestructura * } 100\end{array}$ \\
\hline
\end{tabular}

Fuente: Elaboración propia. 


\section{Conclusiones y consideraciones.}

Mediante la presente aportación, la pretensión en lo general, es que estos indicadores:

1. Sirvan de medios, instrumentos o mecanismos eficaces y eficientes para evaluar hasta qué punto 0 en qué medida se están logrando los objetivos estratégicos institucionales, metas y responsabilidades.

2. Produzcan información pertinente y válida para analizar el desempeño del sistema de investigación institucional en la IES, verificando el cumplimiento de los objetivos en términos de resultados a la productividad, significancia, contribución social y el valor agregado o entregado y reconocido por los clientes.

3. Detecten y prevean desviaciones en el logro de los objetivos y generen alertas sobre la acción para mantener la dirección alineada con respecto al logro de la misión y visión institucional.

Adicionalmente en lo particular, la perspectiva es que preponderantemente:

1. Impulsen los esfuerzos para medir los resultados en el abatimiento de los principales rezagos institucionales y el impacto social de las acciones realizadas en el ámbito de la investigación universitaria.

2. Dispongan de elementos informativos significativos, válidos y pertinentes para fortalecer la planeación, así como para medir, evaluar y apoyar el desempeño institucional, en función de objetivos y metas a partir de indicadores pertinentes y homogéneos que brinden información comparable interinstitucionalmente.

3. Generen e incorporen información sustantiva para apoyar la toma de decisiones con un sentido estratégico y de alto impacto social, permitiendo a su vez que las distintas instancias institucionales puedan identificar con la mayor precisión el grado de cumplimiento de los compromisos asumidos. 


\section{Referencias}

Ávila, A. (2015). Las organizaciones como sistemas sociales complejos. Revista de la Asociación Latinoamericana para la Formación y Enseñanza de la Psicología, 3(9). Recuperado de http://www.integracion-academica.org/10-volumen-1-numero2-2013/25-las-organizaciones-como-sistemas-sociales-complejos.

Bernárdez, M. (2005). Desempeño Humano. Sonora, México: Instituto Tecnológico de Sonora. Recuperado de http://www.expert2business.com/itson/TDHBernardez.pdf

Bernárdez, M. (2005). Desempeño Organizacional. Sonora. México: Instituto Tecnológico de Sonora. Recuperado de http://www.expert2business.com/itson/DOBernardez.pdf

Fayol, H. (1987). Administración Industrial y General. El Ateneo Editorial. Decimocuarta edición. Recuperado de http://isabelportoperez.files.wordpress.com/2011/11/admc3b3n_ind_y_general001. pdf

Franklin, E. (2001): Auditoría Administrativa. México: Editorial Mc. GRAW-HILL.

Foro Consultivo Científico y Tecnológico, AC. (2011). Ranking de Producción Científica Mexicana versión 2011. Recuperado de http://www.foroconsultivo.org.mx/libros_editados/ranking_por_institucion_2011.pdf.

Guerra, I. (2007). Evaluación y Mejora Continua. Conceptos y Herramientas para la Medición y Mejora del Desempeño. Un Enfoque en resultados e impacto. Author House. ITSON. Global Business Press.

González. L. \& Ayarza, H. (1997). Calidad, evaluación institucional y acreditación en la educación superior en la región Latinoamericana y del Caribe. Caracas: CRESALC/UNESCO.

González, O. (2005). Los sistemas de control de gestión estratégica de las Organizaciones. Trabajo 71. Recuperado de www.momografías.com.

Kaufman, R. (2005). Planificación Mega: Herramientas prácticas para el éxito Organizacional. España: Universitat Jaume I.

Lezama, C. (2007). Indicadores de gestión. Recuperado de: http://webcache.googleusercontent.com/search?q=cache:zBf1IENbm4J:www.inegi.org.mx/inegi/spc/doc/bibliografia/indicadores-degestion. $d o c x+\& c d=4 \& h|=e s-419 \& c t=c| n k \& g l=m x$ 
Indicadores de desempeño para investigación universitaria, en el contexto de mega planeación.

José Irigoyen Baldenegro

Ortiz, A. (2005). Alta gerencia educativa: ¿Cómo dirigir y administrar con eficiencia? Centro de Estudios Pedagógicos y Didácticos. Colombia: CEPEDID.

Royero, J. (2003). Gestión de sistemas de investigación en América Latina. Revista Iberoamericana de Educación, OEI, 30,1-19 septiembre-diciembre de 2002. Recuperado de http://www.rieoei.org/deloslectores/412Royero.pdf

Universidad Nacional Autónoma de México - UNAM. (2005). Catálogo de Indicadores de desempeño de entidades y dependencias universitarias. Recuperado de http://www.planeacion.unam.mx/Planeacion/Apoyo/cat_indicadores_2005.pdf. 